7th International Workshop on Astronomy and

Relativistic Astrophysics (IWARA 2016)

International Journal of Modern Physics: Conference Series

Vol. 45 (2017) 1760051 (5 pages)

(C) The Author(s)

DOI: $10.1142 / \mathrm{S} 2010194517600515$

\title{
On the Submergence and Reemergence of Magnetic Fields in Young Supernovae
}

\author{
Cristian G. Bernal \\ Instituto de Matemática, Estatística e Física \\ Universidade Federal de Rio Grande (FURG) \\ Av. Itália km 8 Bairro Carreiros, Rio Grande, Rio Grande do Sul, Brazil \\ bernalcg@gmail.com
}

Published 15 August 2017

\begin{abstract}
In this work I present numerical magnetohydrodynamic (MHD) simulations of the early dynamics around newly born neutrons stars using the AMR Flash method. When the core-collapse supernovae occurs a reverse shock is formed allowing strong accretion onto the neutron star surface (hypercritical phase). In such regime large amounts of matter are deposited on the neutron star surface, submerging the magnetic field in the new crust. When the hypercritical regime is over, the magnetic field can suffer a reemergence episode due to magnetic diffusion processes, allowing the delayed switch-on of pulsars.
\end{abstract}

Keywords: Supernovae, Neutron Star, Magnetohydrodynamics.

PACS numbers: 26.60.-c, 26.60.Gj, 97.10.Cv

\section{Introduction}

It is widely accepted that core-collapse supernovae are ideal laboratories for fundamental astrophysics because they exhibit extreme conditions of density and temperature not found in terrestrial environments. Besides being responsible for the nucleosynthesis of a large fraction of heavier elements than iron, they give birth to neutron stars and stellar black holes as compact remnants. The presence of such strong gravitational field in the heart of the supernova produces a rich and complex plasma-dynamics around the newly formed compact object few seconds after the explosion ${ }^{1}$.

In the stellar core-collapse scenario the residual compact remnant could be a neutron star immersed in a dense environment. Such newly born neutron star may be exposed to an hyperaccretion phase few seconds after the supernova event. When the ejected matter collides with denser material external layers, it is bifurcated and

This is an Open Access article published by World Scientific Publishing Company. It is distributed under the terms of the Creative Commons Attribution 4.0 (CC-BY) License. Further distribution of this work is permitted, provided the original work is properly cited. 
a reverse shock is formed. In that case, some part of ejected material continues on its way to the outside where the hydrodynamics effects are not very important, while another part of the material fallback onto the surface of the proto-neutron star. This reverse shock allows to deposit large amounts of matter onto the protoneutron surface forming a new crust. For the SN1987A case the deposited material was estimated in $\sim 0.15 M_{\odot}$, in two hours of strong accretion ${ }^{2}$.

The analytical models that describe such hypercritical accretion onto neutron star surfaces use several assumptions: stationary flows, spherical symmetry of the flow, ideal gas as equation of state, only pair annihilation processes in the neutrino cooling process, and negligible magnetic field of the compact remnant.

In the present work, we revisit the hypercritical accretion phase onto newly born neutron stars, with better temporal and spatial resolutions in the simulations, focusing on the rich plasma-dynamics and MHD morphology around the newly born neutron star. Besides considering the magnetic field, we are including neutrino cooling due pair production and other neutrino processes as well as a better equation of state in our model. Also, we analyzed the magnetic field evolution just after the hyper-accretion stops. In section 2 we discuss the numerical model used in this work, with all the relevant physics and numerical technique. In section 3 we discuss and interpret the results of our simulations. The relevant references are in section 4 .

\section{Physical Ingredients and Numerical Procedure}

To tackle numerically the hypercritical accretion problem, as was described above, we use a customized version of the Eulerian, multiphysics, parallel Flash Code ${ }^{3}$, which use the Split Eight-Wave solver to solve the whole set of MHD equations. The matter equation of state is an adaptation of Flash's Helmholtz package which includes contributions from the nuclei, $e^{+}-e^{-}$pairs, radiation and the Coulomb correction. This routine contains more physics and is appropriate for addressing astrophysical phenomena in which electrons and positrons may be relativistic and/or degenerate and in which radiation may significantly contribute to the thermodynamic state. Full details of the Helmholtz equation of state are provided in Ref. ${ }^{4}$.

Neutrino energy losses are dominated by the annihilation process, which involves the formation of a neutrino-antineutrino pair when an electron-positron pair is annihilated near the stellar surface $\left(e^{+}-e^{-} \rightarrow \nu+\bar{\nu}\right)$. However, we also include other relevant neutrino processes present in such regime: (i) the photo-neutrino process $\left(\gamma+e^{ \pm} \rightarrow e^{ \pm}+\nu+\bar{\nu}\right)$; (ii) the plasmon decay process $(\gamma \rightarrow \nu+\bar{\nu})$; (iii) and the Bremsstrahlung process $\left(e^{ \pm}+N \rightarrow e^{ \pm}+N+\nu+\bar{\nu}\right)$ or $(N+N \rightarrow N+N+\nu+\bar{\nu})$. All these processes, which are implemented in a customized module in the code, are described in Ref. ${ }^{5}$.

I want to simulate a portion of the surface of the neutron star (spherical geometry) doing a mapping of the surrounding cone above the neutron star into an accreting column (cartesian geometry). This mapping allows me to study the accretion shock in more detail. For the 2-dimensional computational domain I consider 
wide columns with a base of $\Delta x=2 \times 10^{6} \mathrm{~cm}$, centered on $x=0$, and a height $\Delta y=10^{7} \mathrm{~cm}$, with $y=10^{6} \mathrm{~cm}$ being the neutron star radius. The gravitational acceleration (plane-parallel external field) is taken as $g_{y}=-G M / y^{2}$ and we assume a standard neutron star mass of $1.4 M_{\odot}$. In order to study the evolution of the magnetic field we take as magnetic initial condition a magnetic field loop, in the shape of an hemi-torus.

On the central hemi-circle of the loop the field has a strength $B_{0}=10^{12} \mathrm{G}$ (standard for neutron stars) and about it is shaped as a Gaussian profile, where the thickness of the loop is $R=1 \mathrm{~km}$ and the two feet of the loop are centered at $x=-5$ and $x=+5 \mathrm{~km}$. The lateral boundaries are outflow-type and the top boundary is inflow with free fall conditions for velocity and accretion rate, as Ref. ${ }^{6}$. At the bottom, on the neutron star surface, we use a custom boundary condition that enforces the hydrostatic equilibrium. For the magnetic field, the two lateral sides are also treated as periodic boundaries, while at the bottom the field is frozen from the initial condition, i.e., the two feet of the loop are anchored into the neutron star and no field can be pushed into the star by the accretion.

\section{Results}

I am interested in following the evolution of the magnetic field, which is anchored onto the proto-neutron star surface, when the hypercritical accretion phase take place. The accretion rates per area $\dot{m}$, considered here will be expressed in terms of a fiducial rate

$$
\dot{m}_{0}=1.75 \times 10^{15} \mathrm{~g} \mathrm{~cm}^{-2} \mathrm{~s}^{-1},
$$

and always assumed to be constant during the entire simulations of the hypercritical phase. This corresponds to a total accretion rate onto the neutron star of

$$
\dot{M}_{0} \sim 350 M_{\odot} \mathrm{yr}^{-1},
$$

which is the accretion rate originally estimated by Ref. ${ }^{2}$ for SN1987A. Here, the goal was to find the complete submergence of the magnetic field and the size of the new crust. Due this, the simulations has good time resolution and excellent spatial resolution.

I start the simulation just before the reverse shock reaches the magnetic loop, which is initially immersed in a low density medium $\left(\rho \simeq 10^{2} \mathrm{~g} \mathrm{~cm}^{-2}\right)$, and above it the falling-back matter has a free-fall velocity and a density obtained by mass conservation:

$$
v_{\mathrm{ff}}=\sqrt{2 G M / y} \text { and } \rho_{\mathrm{ff}}=\dot{m} / v_{\mathrm{ff}} .
$$

In Fig. 1 we show color maps of density (on a logarithmic scale) and the magneticRAM pressures ratio (on a linear scale), for several weakly hyper-accretion rates with superimposed magnetic field lines: $\dot{m}=(0.1,0.01,0.001) \times \dot{m}_{0}$. The timescales showed are $t=(100,400,700) \mathrm{ms}$ respectively, when a quasi-hydrostatic envelope 

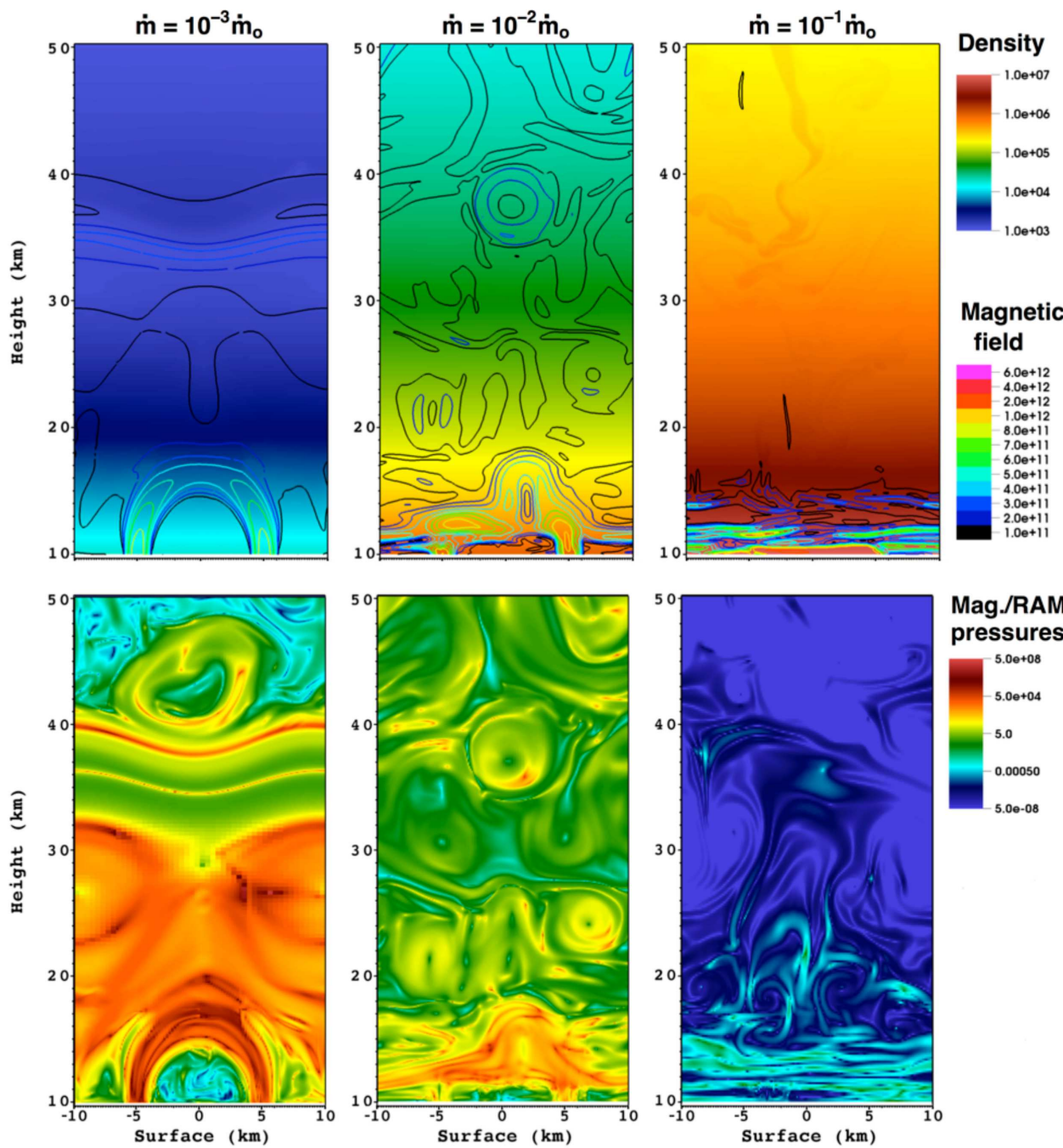

Fig. 1. Color maps of density and magnetic-RAM pressures ratio for several accretion rates: $\dot{m}=(0.1,0.01,0.001) \times \dot{m}_{0}$. The timescales showed here correspond to $t=(100,400,700) \mathrm{ms}$, from the highest to the lower accretion rates, respectively. We show simulations when a quasi-hydrostatic envelope has been almost established.

is almost established. Here, the RAM pressure is defined as $P_{R A M}=\rho v^{2}$ and the magnetic pressure is, basically, $P_{B}=B^{2} / 8 \pi$. This phase is active for $3-2$ hours, and the material piled up by hyper-accretion builds a new stellar crust, submerging the magnetic field within.

At this point, the magnetic loop looks more flattened due to the high accretion over it. The bulk magnetic field is confined into the new crust, although small residual magnetic fields float within the quasi-hydrostatic envelope without affecting 
the dynamics. Moreover, depending on the strong accretion rate, the submerged magnetic field could be pushed into the mantle, where it will be crystallized.

To analyze the possible re-diffusion of some portion of the submerged magnetic field, immediately after the hypercritical phase stops, we started the simulations with the quasi-hydrostatic structure including the new formed crust with the magnetic field immersed inside. The submerged magnetic field suffer magnetic reconnection episodes within the new crust too, allowing some portion of the magnetic field is disconnected leaving the crust by buoyancy effects or MHD instabilities. The remain portion of the magnetic field can be crystallized and pushed into the neutron star mantle. This suggests that neutron stars produced by supernovae, in which post core-collapse accretion is hypercritical, are born with a weak superficial magnetic field.

\section{References}

1. A. Mezzacappa, Neutrinos and Neutrino Physics: Still Center Stage in Core Collapse Supernovae, in APS April Meeting Abstracts, p. 1002, Apr. (2006).

2. R. A. Chevalier, Neutron Star Accretion in a Supernova, APJ 346, 847 (1989).

3. B. Fryxell, K. Olson, P. Ricker, F. X. Timmes, M. Zingale, D. Q. Lamb, P. MacNeice, R. Rosner, J. W. Truran, and H. Tufo, FLASH: An Adaptive Mesh Hydrodynamics Code for Modeling Astrophysical Thermonuclear Flashes, APJS 131, 273 (2000).

4. F. X. Timmes and F. D. Swesty, The Accuracy, Consistency, and Speed of an ElectronPositron Equation of State Based on Table Interpolation of the Helmholtz Free Energy, APJS 126, 501 (2000).

5. N. Itoh, H. Hayashi, A. Nishikawa, and Y. Kohyama, Neutrino Energy Loss in Stellar Interiors. VII. Pair, Photo-, Plasma, Bremsstrahlung, and Recombination Neutrino Processes, APJS 102, 411 (1996).

6. C. G. Bernal, D. Page, and W. H. Lee, Hypercritical Accretion onto a Newborn Neutron Star and Magnetic Field Submergence, APJ 770, 106 (2013). arXiv:1212.0464](2012). 